\title{
Robustness tests provide further support for an ecological account of the truth and fake news by repetition effects
}

\author{
Jérémy Béna ${ }^{1}$, Olivier Corneille ${ }^{1}$, Adrien Mierop $^{1}$, \& Christian Unkelbach ${ }^{2}$ \\ ${ }^{1}$ UCLouvain, Belgium \\ ${ }^{2}$ Social Cognition Center Cologne, University of Cologne, Germany
}

\section{[Preprint]}

Word count: 2994 (without the title page, references, footnotes, and figure captions)

Running title: Replication studies of Corneille et al. (2020)

Conflict of interest statement: The authors declare no conflict of interest.

Funding: This research did not receive any specific grant from funding agencies in the public, commercial, or not-for-profit sectors.

Correspondence concerning this article should be addressed to Jérémy Béna, UCLouvain, PSP IPSY, 10 Place du Cardinal Mercier, 1348, Louvain-la-Neuve, Belgium. Email:

jeremy.bena@uclouvain.be

\section{CRediT authorship contribution statement}

JB: Conceptualization, Data curation, Formal analysis, Investigation, Methodology, Software, Visualization, Writing - original draft. OC: Methodology, Resources, Writing - review \& editing. AM: Methodology, Resources, Writing - review \& editing. CU: Methodology, Resources, Writing - review \& editing. 


\begin{abstract}
Corneille et al. (2020) found that repetition increases judgments that statements have been used as fake news on social media, a result that is consistent with an ecological theorization. They also found that repetition increases truth judgments and decreases falsehood judgments (i.e., two instantiations of the Truth-by-Repetition effect), which is more compatible with the ecological account than with competing accounts. However, the first author of the present article found unsuspected programming issues in Corneille et al.'s experiments. These programming issues introduced confounds that may have been responsible for the results. To estimate whether Corneille et al.'s results and main claims hold when correcting these issues, the current team agreed on two high-powered preregistered replications of Corneille et al.'s experiments $\left(N_{\text {total }}=\right.$ 540). The findings strongly support Corneille et al.'s predictions, which substantiates and generalizes an ecological account of repetition effects on judgment.
\end{abstract}

Keywords: ecological account; fakeness-by-repetition; processing fluency; repetition; truth-byrepetition; truth effect 


\section{Robustness tests provide further support for an ecological account of the truth and fake news by repetition effects}

\section{Introduction}

Being repeatedly exposed to the same piece of information typically increases its perceived truth (for meta-analysis, see Dechêne et al., 2010; for overviews, see Brashier \& Marsh, 2020; Unkelbach et al., 2019). A prominent explanation for the "Truth-by-Repetition" (TBR) effect is processing fluency, as repeated exposure to information makes the information's processing more fluent (e.g., Feustel et al., 1983). There are three accounts why processing fluency due to repetition might increase people's subjective truth judgments. First, fluency is a positive experience (e.g., Winkielman et al., 2003), and as truth is also positively connotated, the positive experience might amplify truth judgments. Second, fluency might amplify any judgment (Albrecht \& Carbon, 2014, Landwehr \& Eckmann, 2020). And third, fluency might be a valid ecological cue for truth (e.g., Reber \& Unkelbach, 2010)

For the latter point, the information ecology is critical to people's interpretation of fluency (e.g., Unkelbach \& Greifeneder, 2013). For instance, if the TBR effect is typically observed when the ecology is left unspecified, this would be because truth is more common than falsehood in people's regular ecologies. In contexts where fake news spreads widely, however, such as social media (Del Vicario, 2016; Vosoughi et al., 2018; but see Juul \& Ugander, 2021), an ecological model predicts that the repetition-induced fluency would be a cue for "fake news" judgments.

Corneille et al. (2020) recently tested these three explanations against each other. Across three experiments, they found evidence consistent with the ecological account. The strongest 
point relied on two preregistered studies (Experiments $1 \& 2$ ). In these experiments, participants read statements in an exposure phase (e.g., "Babies have more bones than adults"). These statements were then and present again intermixed with new ones (i.e., that were not displayed in the exposure phase) in a judgment task. Critically, participants did not indicate the statements' truth, but whether the statements have been previously used as fake news on social media. Corneille et al. found that repeated statements were more likely to be perceived as used as fake news on social media than new statements. As fake news is clearly negatively connotated, this pattern does not align with a "fluency is positive" hypothesis. In a third preregistered study, Corneille et al. (Experiment 3), participants judged either the "falsehood" or the "truth" of repeated and new statements in an unspecified ecology. The data showed that repetition significantly increases perceived truth and significantly decreases perceived falsehood (although to a lesser extent) - a result that is incompatible with an amplification account, which would predict an increase in both conditions. Thus, the ecological account was the only one that predicted the data pattern of all experiments. Corneille et al.'s results are important theoretically (they help separate between competing fluency accounts) and practically (they may help predict the direction in which repetition influences judgments according to the information ecology).

However, there are concerns regarding the procedural details of the reported Experiments 1 and 2, which carry the brunt of the theoretical arguments. Reanalyses of Corneille et al.'s publicly shared data point to programming issues that caused significant deviations from the original, intended procedure ${ }^{1}$. Specifically, (1) some repeated statements were displayed more

\footnotetext{
${ }^{1}$ We strongly suspect the programming issues originate in Python-to-Javascript conversion problems applying to the version of the OpenSesame/OSWeb program used in Corneille et al.'s studies. The issues went unnoticed by the authors because the R program designed and pretested for the analyses correctly ran on the data output generated by
} 
than once in the exposure phase, resulting in (2) more new than repeated statements in the truth judgment task, (3) only part of the responses from Experiments 1 and 2's judgment phase was correctly encoded by the program. Reanalyses further indicated that factual truth was programmed to be orthogonal to repetition on the aggregate but not on the individual level. As a further concern, this randomization failed to be achieved also on the aggregated level ${ }^{2}$.

These deviations are problematic because Corneille et al. (2020) aimed at using a typical TBR paradigm (see Unkelbach et al., 2019). In most TBR studies using one repetition, (1) factual truth is orthogonal to repetition, (2) at the participant, not aggregated level, (3) each repeated statement is encountered exactly once in the exposure phase, and (4) there are as many repeated and new statements in the truth judgment task. As a result, it is unknown whether testing Corneille et al.'s hypotheses in a more controlled, typical TBR paradigm would yield the same evidence for their claims and, as a result, for the ecological account they were based on.

To test the robustness of the effects Corneille et al. (2020) found, we conducted close high-powered and preregistered replications of their Experiment 2 (which was a higher-powered replication of their Experiment 1) and Experiment 3 after fixing the abovementioned programming issues. We expected to replicate the support for the ecological account and predicted that repeated statements would be more often categorized as "Yes, used as Fake News on social media" than new statements (Experiment 1) and that repetition would increase perceived truth but decrease perceived falsehood (Experiment 2). Should this be the case, this

the online platform. Another issue was inherent in the programmation itself, which sought randomization of statements' factual truth at the aggregated level (and failed to achieve it because of the abovementioned conversation issue) rather than participants' level. More information on the issue is available at https://osf.io/asjfr/.

${ }^{2}$ The reanalyses are available at https://osf.io/gzdta/. 
would not just support conclusions reached in the original article but also support the robustness of these conclusions across testing conditions that substantially differ from each other.

We report how we determined our sample size, all data exclusions, all manipulations, and all measures in the two replication studies. The preregistration, experiment programs, data, and analyses are publicly available at https://osf.io/qzeas/.

\section{Experiment 1: Replication of Corneille et al.'s Experiment 2}

\subsection{Participants and design}

The design was a 2 (Repetition: Repeated vs. New) $\times 2$ (Factual truth: True vs. False), with the two factors manipulated within participants.

Based on the sample size of Corneille et al. (2020, Experiment 2, $N=152$ ), we aimed for 160 participants. A power analysis indicated that a sample of $N=160$ gives us ample power to find the main effect of repetition by Corneille et al. as statistical power of $1-\beta=.95$ was achieved with only $N=52.160$ participants completed the experiment on Prolific (65.63\% female, seven not reported; $M_{a g e}=31.75, S D_{a g e}=12.37$, one not reported). Participants were English speakers (as per Corneille et al., 2020), and they did not take part in previous TBR effect-related studies we conducted. Participants were paid US \$ 1.02 for completing the study.

\subsection{Materials and procedure}

Instructions and materials were identical to Corneille et al. (2020, Experiments $1 \& 2)$. After providing their informed consent, participants first entered the exposure phase, with the instruction to read 20 statements (10 true; 10 false, randomly selected from a list of 20 true and 20 false statements). Statements were sequentially displayed in the middle of the screen for 2500 milliseconds (inter-trial time: 1000 milliseconds). The statements displayed in the exposure phase were the "repeated" statements. Next, participants entered the judgment phase, where the 
20 repeated statements were mixed with 20 new statements (half true) and displayed in a random order. Participants indicate whether yes (by pressing the "y" key) or no (by pressing the "n" key) each statement "has been previously used as a Fake News on the social media." Participants were then thanked and debriefed. The complete instructions are available in Corneille et al.'s (2020) preregistration of Experiment 1 (https://osf.io/hsv9y/) and in the program of the present experiment. We programmed the experiment with lab.js (Henninger et al., 2021), and we used JATOS (Lange et al., 2015) to run the study online on Prolific.

The critical differences between the present replication study and Corneille et al.'s methodology is that (1) all participants saw as many true and false statements in each repetition condition (vs. less factually false repeated statements than other statements in Corneille et al.); (2) all repeated statements were repeated only once (vs. some statements being selected more than one time in the exposure phase in Corneille et al.), and (3) participants judged the same number of repeated and new statements (vs. more new than repeated statements in Corneille et al.).

\subsection{Results}

We analyzed the data with a general linear model using the 'lme4' R package (Bates et al., 2015). Our dependent was the proportion of "yes, used as Fake News on social media" judgments. Repetition (Repeated vs. New) and Factual truth (True vs. False) were the factors, and participants were entered as a random effect. 


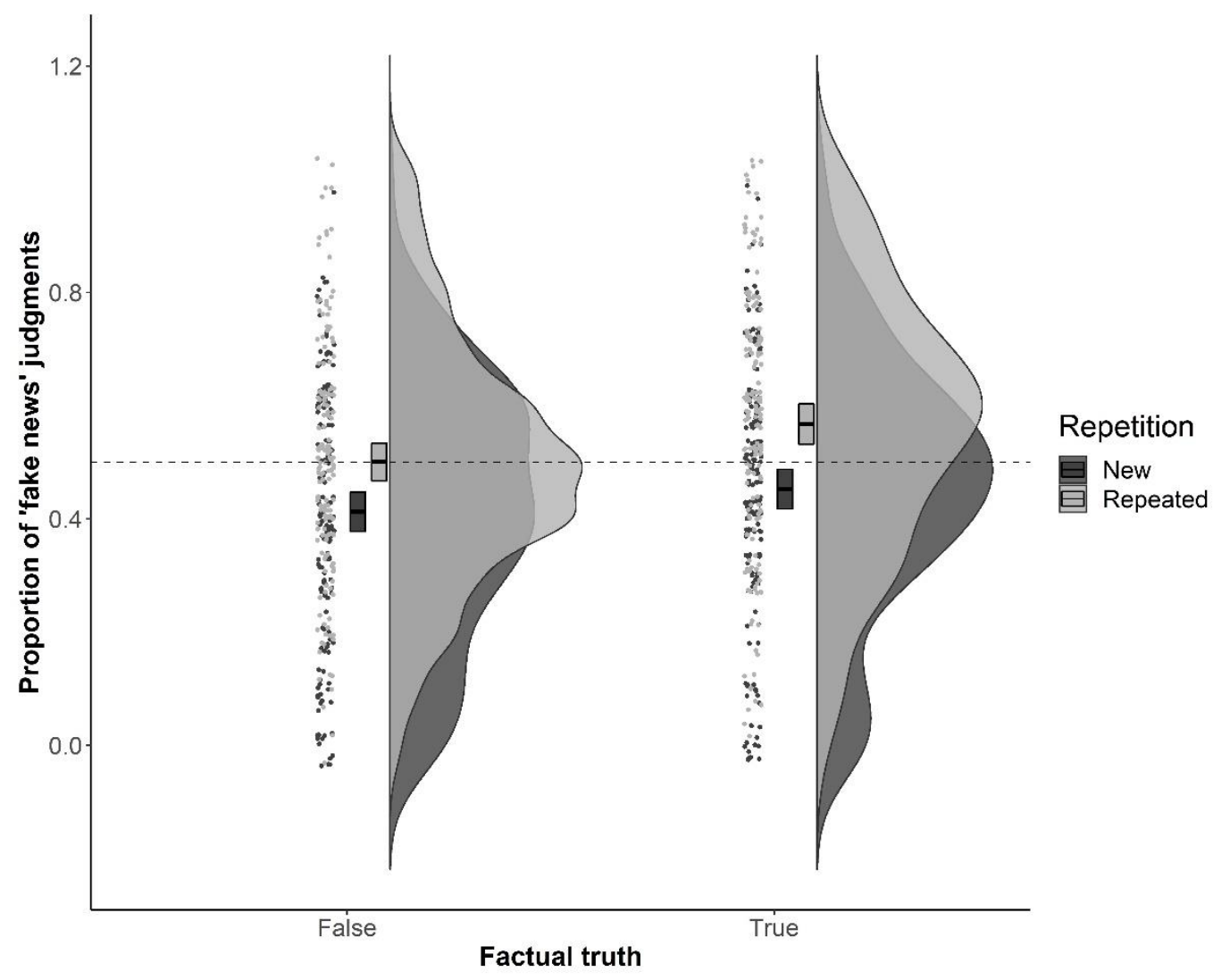

Figure 1. Proportions of "fake news" judgments as a function of Repetition (dashed horizontal line: no bias toward the "yes, fake news" or the "no, not fake news" side) and Factual truth in Experiment 1. The dots are participants' scores (jittered). The lower and upper limits of the boxplots are the $95 \%$ confidence intervals, with the mean in between. The distributions are the kernel probability density of the data.

We replicated the results by Corneille et al. (2020): The proportion of "yes, used as Fake News on social media" judgments was larger for Repeated $(M=.53 ; S D=.2)$ than new $(M=.43$; $S D=.21$ ) statements, $F(1,477)=47.36, p<.001$ (see Figure 1). Contrary to Corneille et al.'s findings, the effect of factual truth was also significant, $F(1,477)=13.11, p<.001$ : The proportion of "yes, used as Fake News on social media" judgments was larger for true ( $M=.51$; $S D=.17)$ than false $(M=.46 ; S D=.16)$ statements, replicating a finding by Unkelbach and Stahl 
(2009). The interaction between Repetition and Factual truth was not significant, $F(1,477)=$ $0.83, p=.363$

\subsection{Discussion}

Experiment 1 replicates Corneille et al.'s (2020) experiments 1 and 2: we found the "fake news by repetition effect": Participants perceived repeated statements more likely to be "fake news" than new statements. Thus, in line with an ecological account of the TBR effect, and contradicts a "fluency is positive" explanation. In information ecologies where fake news is are likely to be repeated, fluency may be used as a cue for perceived fake news.

However, in the present experiment (and Experiments 1 and 2 of Corneille et al., 2020), an amplification account makes the same prediction, as fluency might amplify judgments on any dimension; whether it is truth, falsehood, or fakeness). Thus, Corneille et al. designed

Experiment 3, asking participants to judge the truth or falsehood of repeated and new statements; an amplification account predicts a main effect of repetition, an ecological account an interaction of judgment and repetition. Their Experiment 3 was thus critical but had similar programming issues as Experiments 1 and 2. We thus replicated Corneille et al.'s Experiment 3.

\section{Experiment 2: Replication of Corneille et al.'s Experiment 3}

\subsection{Participants and design}

The design was a 2 (Repetition: Repeated vs. New) $\times 2$ (Factual truth: True vs. False $) \times 2$ $\times 2$ (Judgment: Judge truth vs. Judge falsehood), with the two first factors manipulated within participants and the last factor manipulated between participants.

380 participants completed the experiment on Prolific $(65.26 \%$ female, seven not reported; $M_{\text {age }}=31.99, S D_{\text {age }}=11.47$, one not reported; $n=199$ in the Judge truth condition and 
$n=181$ in the Judge falsehood condition). Participants were English speakers and did not take part in TBR effect-related studies we conducted, as per Corneille et al. (2020). Participants were paid US \$ 0.74 for completing the study.

We used the summary statistics by Corneille et al. in their Experiment 3 to estimate power; the R script of the power analyses is available at https://osf.io/xmb2a/. The power analyses indicated that a sample of $N=380$ ( $n=190$ in each Judgment condition) gives us sufficient power to find the simple effect of Repetition Corneille et al. found in the "falsehood" condition, which is our smallest effect of interest here. We thus aimed for a sample size of 380 participants; the original sample size in Experiment 3 was $N=200$.

\subsection{Materials and procedure}

Instructions and materials were identical to Corneille et al. (2020, Experiment 3) and highly similar to the present Experiment 1, with the following deviations: Participants were randomly allocated to one of the two Judgment conditions (Judge truth or Judge falsehood) in the judgment phase. Participants indicated whether yes (by pressing the "y" key) or no (by pressing the " $n$ " key) each statement is true (Judge truth condition) or false (Judge falsehood condition). The complete instructions are available in Corneille et al.'s (2020) preregistration of Experiment 3 (https://osf.io/bvfy9/) and in the program of the present experiment (see Experiment 1 for details). 


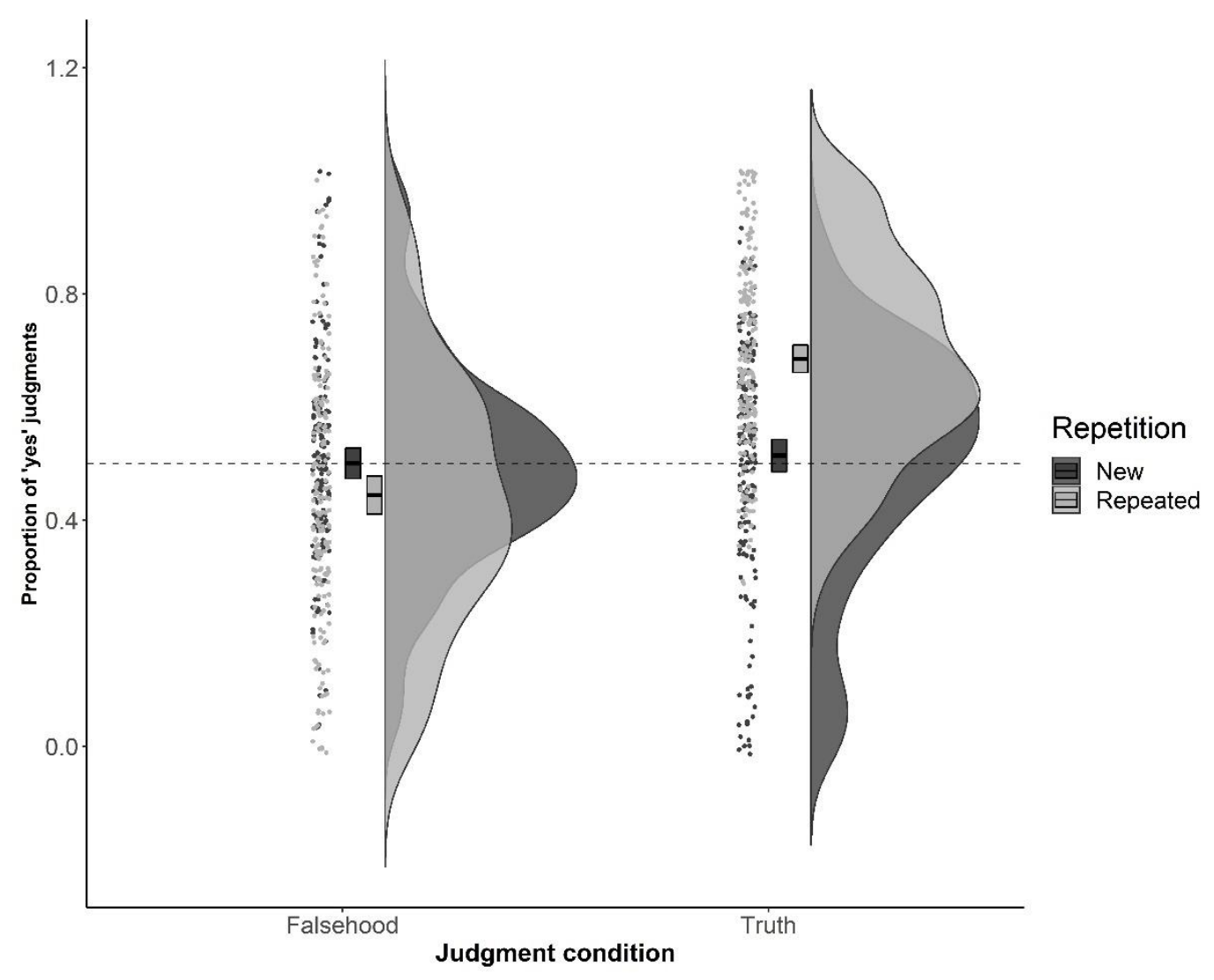

Figure 2. Proportions of "yes" judgments as a function of Repetition (dashed horizontal line: no bias toward the "yes" or the "no" side) and Judgment condition in Experiment 2. The dots are participants' scores (jittered). The lower and upper limits of the boxplots are the $95 \%$ confidence intervals, with the mean in between. The distributions are the kernel probability density of the data.

\subsection{Results}

As done by Corneille et al. (2020), we analyzed the proportion of "yes" judgments as a function of Repetition (within participants) and Judgment (between participants) in a mixed ANOVA.

As predicted and replicating Corneille et al., we found a significant interaction between Repetition and Judgment on the proportions of "yes" judgments, $F(1,378)=50.04, p<.001, \eta^{2}$ p $=.12$ (see Figure 2). Repetition increased judgments of truth (Judge truth condition) but 
decreased judgments of falsehood (Judge falsehood condition). In the Judge Truth condition, participants judged repeated statements more often as true $(M=.69 ; S D=.17)$ than new statements $(M=.51 ; S D=.2), t(198)=-8.16, p<.001, d=0.907$. We found the reversed effect in the Judge Falsehood condition; participants judged repeated statements less often as false ( $M$ $=.44 ; S D=.23)$ than new statements $(M=.5 ; S D=.18), t(180)=2.29, p=.023, d=0.269$.

As the two simple effects of Repetition on proportions of "yes" judgments went in opposite directions in each Judgment condition, we compared the proportions of "yes, true" (Judge truth condition) and the proportions of "no, not false" (i.e., true - Judge falsehood). In doing so, we were able to estimate whether the two simple effects differ in magnitude besides going in opposite directions. As suggested above by the magnitude of the Cohen's $d$ of the two simple effects of Repetition, the effect of Repetition was larger in the Judge truth condition than in the Judge falsehood condition, $F(1,378)=12.83, p<.001, \eta^{2} \mathrm{p}=.03$.

\subsection{Discussion}

We fully replicated Corneille et al.'s Experiment 3: Repetition increased perceived truth and decreased perceived falsehood, contradicting the amplification account for which repetition should have increased both perceived truth and falsehood.

\section{General Discussion}

We fully replicated all results in Corneille et al.'s (2020) experiments: We found that repetition increased the likelihood that statements are perceived as fake news in a social media information ecology (Experiment 1). When the information ecology was left unspecified, repetition increased perceived truth and, to a smaller extent, decreased perceived falsehood (Experiment 2). These results are in line with an ecological explanation of fluency effects (Unkelbach, 2006, 2007; Unkelbach \& Greifeneder, 2013) and do not support an amplification 
account (Albrecht \& Carbon, 2014) or "fluency-is-positive" explanation (e.g., Winkielman et al., 2003).

One interesting finding, deviating from Corneille et al., was that participants perceived factually true statements more likely as fake news than false statements, independent of repetition (Experiment 1). It is possible that making factual truth and repetition orthogonal for all participants (where Corneille et al. had more factually true repeated than factually true statements at the aggregated level) increased the likelihood to detect the effect of factual truth. In any case, this main effect of statements' factual truth suggests that, at least in the present sample, participants had some previous knowledge on the statements - a prerequisite condition for factual truth to be a psychologically meaningful factor.

It may seem counterintuitive that participants perceived true statements as more likely to be fake news on social media. A fluency explanation, however, accounts for this result. For instance, Unkelbach and Stahl (2009, Experiment 2) found that, regardless of repetition, true statements were more likely to be judged as false when participants learned that all statements were false in the exposure phase. This follows because true statements were likely encountered before, but not false ones. As a result, true statements and repeated statements would be more fluently processed, which indicates falseness when all repeated statements are false. Applying this reasoning to the present experiment, true statements might have been more fluently processed than false ones, resulting in truth-induced fluency being used as a cue in line with the judgment's instructions; i.e., responding that statements were used as fake news on social media.

Beyond these interesting findings, the present results show the robustness of Corneille et al.'s (2020) findings. As psychology is characterized by a "credibility revolution" (Vazire, 2018) in which replication studies often call into question past conclusions even using designs very close 
to the ones of the original studies (e.g., Camerer et al., 2018; Open Science Collaboration, 2015), we believe that it is not trivial to find consistent support for a theory (the ecological account) across experiments that significantly differ from each other. Reporting not just replication failures but also replication successes is a crucial endeavor to collectively gain scientific discernment (i.e., to discriminate true and false effects better). By confirming and generalizing results and conclusions from Corneille et al., the findings of the present two replication studies provide converging support for the ecological theorization of repetition effects on truth judgments.

\section{Conclusion}

Two preregistered studies fully replicated the effects reported by Corneille et al. (2020). The replications were motivated by programming issues in the original experiments discovered after they were published. The present studies show that Corneille et al.'s results were not artefactually created by the specific designs implemented. Rather, their effects and conclusions hold in the more controlled designs we used here. As a result, the ecological account, for which fluency's interpretation depends on the judgment context, comes out further supported. 


\section{References}

Albrecht, S., \& Carbon, C. C. (2014). The fluency amplification model: Fluent stimuli show more intense but not evidently more positive evaluations. Acta Psychologica, 148, 195-203. https://doi.org/10.1016/j.actpsy.2014.02.002

Bacon, F. T. (1979). Credibility of repeated statements: Memory for trivia. Journal of Experimental Psychology: Human Learning and Memory, 5(3), 241252. https://doi.org/10.1037/0278-7393.5.3.241

Bates, D., Maechler, M., Bolker, B., \& Walker, S. (2015). Fitting Linear Mixed-Effects Models Using lme4. Journal of Statistical Software, 67(1), 1-48. https://doi.org/10.18637/jss.v067.i01

Brashier, N. M., \& Marsh, E. J. (2020). Judging Truth. Annual Review of Psychology, 71(1), 499-515. https://doi.org/10.1146/annurev-psych-010419-050807

Camerer, C. F., Dreber, A., Holzmeister, F., Ho, T. H., Huber, J., Johannesson, M., Kirchler, M., Nave, G., Nosek, B. A., Pfeiffer, T., Altmejd, A., Buttrick, N., Chan, T., Chen, Y., Forsell, E., Gampa, A., Heikensten, E., Hummer, L., Imai, T., Isaksson, S., ... Wu, H. (2018). Evaluating the replicability of social science experiments in Nature and Science between 2010 and 2015. Nature human behaviour, 2(9), 637-644. https://doi.org/10.1038/s41562-018-0399-z

Corneille, O., Mierop, A., \& Unkelbach, C. (2020). Repetition increases both the perceived truth and fakeness of information: An ecological account. Cognition, 205, 104470.

https://doi.org/10.1016/j.cognition.2020.104470 
Dechêne, A., Stahl, C., Hansen, J., \& Wänke, M. (2010). The Truth About the Truth: A MetaAnalytic Review of the Truth Effect. Personality and Social Psychology Review, 14(2), 238257. https://doi.org/10.1177/1088868309352251

Del Vicario, M., Bessi, A., Zollo, F., Petroni, F., Scala, A., Caldarelli, G., ... Quattrociocchi, W. (2016). The spreading of misinformation online. Proceedings of the National Academy of Sciences, 113(3), 554-559. https://10.1073/pnas.1517441113

Feustel, T. C., Shiffrin, R. M., \& Salasoo, A. (1983). Episodic and lexical contributions to the repetition effect in word identification. Journal of Experimental Psychology: General, 112(3), 309-346.

Hasher, L., Goldstein, D., \& Toppino, T. (1977). Frequency and the Conference of Referential Validity. Journal of Verbal Learning and Verbal Behavior, 16(1), 107-112.

Henninger, F., Shevchenko, Y., Mertens, U. K., Kieslich, P. J., \& Hilbig, B. E. (2021). lab.js: A free, open, online study builder. Behavior Research Methods. https://doi.org/10.3758/s13428-019-01283-5

Henderson, E. L., Westwood, S. J., \& Simons, D. J. (2021). A reproducible systematic map of research on the illusory truth effect. Psychonomic Bulletin \& Review. https://doi.org/10.3758/s13423-021-01995-w

Juul, J. L., \& Ugander, J. (2021). Comparing information diffusion mechanisms by matching on cascade size. Proceedings of the National Academy of Sciences, 118(46), e2100786118, https://doi.org/10.1073/pnas.2100786118

Lakens, D., \& Caldwell, A. R. (2021). Simulation-Based Power Analysis for Factorial Analysis of Variance Designs. Advances in Methods and Practices in Psychological Science, 4(1), 1-14. https://doi.org/10.1177/2515245920951503 
Landwehr, J. R., \& Eckmann, L. (2020). The nature of processing fluency: Amplification versus hedonic marking. Journal of Experimental Social Psychology, 90, 103997. https://doi.org/10.1016/j.jesp.2020.103997

Lange, K., Kühn, S., \& Filevich, E. (2015). Correction: “Just Another Tool for Online Studies” (JATOS): An Easy Solution for Setup and Management of Web Servers Supporting Online Studies. PLOS ONE, 10(7), e0134073. https://doi.org/10.1371/journal.pone.0134073

Open Science Collaboration. (2015). Estimating the reproducibility of psychological science. Science, 349(6251), 1-8. https://doi.org/10.1126/science.aac4716

Reber, R., \& Unkelbach, C. (2010). The epistemic status of processing fluency as source for judgments of truth. Review of Philosophy and Psychology, 1(4), 563-581. https://doi.org/10.1007/s13164-010-0039-7

R Core Team (2021). R: A language and environment for statistical computing. R Foundation for Statistical Computing, Vienna, Austria. URL: https://www.R-project.org/.

Vosoughi, S., Roy, D., \& Aral, S. (2018). The spread of true and false news online. Science, 359(6380), 11461151. https://doi.org/10.1126/science.aap9559

Unkelbach, C. (2006). The learned interpretation of cognitive fluency. Psychological Science, 17(4), 339-345. https://doi.org/10.1111/j.1467-9280.2006.01708.x

Unkelbach, C. (2007). Reversing the truth effect: Learning the interpretation of processing fluency in judgments of truth. Journal of Experimental Psychology: Learning, Memory, and Cognition, 33, 219-230. https://doi.org/10.1037/0278-7393.33.1.219

Unkelbach, C., \& Greifeneder, R. (2013). A general model of fluency effects in judgment and decision making. In C. Unkelbach \& R. Greifeneder (Eds.), The experience of 
thinking: How the fluency of mental processes influences cognition and behaviour (pp. 11-32). New York: Psychology Press.

Unkelbach, C., Koch, A., Silva, R. R., \& Garcia-Marques, T. (2019). Truth by Repetition: Explanations and Implications. Current Directions in Psychological Science, 28(3), 247253. https://doi.org/10.1177/0963721419827854

Unkelbach, C., \& Stahl, C. (2009). A multinomial modeling approach to dissociate different components of the truth effect. Consciousness and Cognition, 18(1), 22-38. https://doi.org10.1016/j.concog.2008.09.006

Vazire, S. (2018). Implications of the credibility revolution for productivity, creativity, and progress. Perspectives on Psychological Science, 13, 411-417. https://doi.org/10.1177/1745691617751884

Winkielman, P., Schwarz, N., Fazendeiro, T. A., \& Reber, R. (2003). The hedonic marking of processing fluency: Implications for evaluative judgment. In J. Musch \& K. C. Klauer (Eds.), The psychology of evaluation: Affective processes in cognition and emotion (pp. 189-217). Lawrence Erlbaum Associates Publishers. 